\title{
Emergencias más frecuentas en animales silvestres
}

\author{
Mauricio Jiménez Soto ${ }^{1} \bowtie$, Karen Vega Benavides ${ }^{1}$, Mauricio Pereira Mora² ${ }^{2}$, Isabel Hagnauer ${ }^{1}$
}

1 Hospital de Especies Menores y Silvestres, Universidad Nacional. Email: drmjimenezsoto@hotmail.com, isabel.hagnauer.barrantes@una.cr, karenluvega@hotmail.com

2 Diagnóstico, control de enfermedades y manejo de animales silvestres. Email. matiscr@gmail.com

En Costa Rica los animales silvestres son de dominio público, sin embargo, el Estado no cuenta con recursos económicos ni el personal capacitado para atender las emergencias en animales de vida libre. Por ello el proyecto Diagnóstico, control de enfermedades y manejo de animales silvestres, del Hospital de Especies Menores y Silvestres (HEMS), de la Escuela de Medicina Veterinaria, ha brindado apoyo a instituciones como MINAE, ONGS y otros, con la finalidad de brindar la atención requerida.

El objetivo de este estudio fue, hacer una valoración de las lesiones más frecuentes en un grupo de 100 animales silvestres de vida libre que ingresaron al HEMS o que requirieron su atención y seguimiento, en un periodo de un año.

Estos pacientes fueron 40 aves, en su mayoría psitácidas, 25 primates principalmente congos, ocho felinos, diez perezosos, cinco venados, diez reptiles, un oso hormiguero y un puercoespín.

De estos casos se seleccionaron cuatro para representar las dificultades de tratamientos y manejo según la especie, además de cómo valorar su pronóstico y las decisiones que se deben tomar con el fin de brindar bienestar animal.

El primer caso es fractura en un felino silvestre al que se le coloco una fijación esquelética externa, el siguiente caso fue la lesión en un ave de vida libre, con su posterior recuperación. Otro de los casos es el manejo y control de una lesión por accidente en un primate y por último, el manejo y seguimiento de una electrocución en un perezoso.

Es importante, crear protocolos y fondos adecuados para su atención, incluyendo equipos multidisciplinarios y de esta manera, mejorar la supervivencia y actuar sobre los factores que desencadenan muchas de estas lesiones.

Autor para correspondencia Mauricio Jiménez Soto: drmjimenezsoto@hotmail.com 